\title{
On the State Estimation for Stochastic Distributed Parameter Systems with Dynamic Boundary Conditions
}

\author{
Masaaki Ishikawa and Hidefumi Hashimoto \\ Faculty of Engineering, Yamaguchi University \\ Tokiwadai, Ube 755 JAPAN \\ Tel : +836359482 Fax : +836290053 \\ e-mail :ishikawa@csse.yamaguchi-u.ac.jp
}

\begin{abstract}
In hydraulic problems, we often encounter nonlinear problems in porous media, for example, dam and percolation problems [1], [2]. Such problems are simply called the filtration problem. This paper is concerned with the state estimation in the filtration problem under the noisy observation. First, the mathematical model of the system dynamics is given and the existence of the unique solution of the system equation is proved. Secondly, based on the noisy boundary observation, the estimator dynamics is derived by using the nonlinear estimation theory and the approximation technique for the nonlinear estimator dynamics is proposed. Finally, the efficiency of the approximation is shown through the numerical example.
\end{abstract}

\section{Introduction}

The characteristics of the filtration problem considered in this paper is that time evolution of the system state at the boundary is governed by the partial differential equation and the boundary condition contains the partial differential inequality. In Section 2, the mathematical model of the filtration problem is given including an explanation of the boundary condition. The mathematical security of the system model is guaranteed by using Lion's technique [3] in Section 3. Section 4 is devoted to deriving the estimator dynamics under the noisy boundary observation and the method to generate a realizable estimator dynamics is introduced. In Section 5, some results of the digital simulation are shown.
Let $(\Omega, \mathcal{F}, \mathcal{P})$ and $\mathcal{F}_{t}, t \in \bar{\Theta}=[0, T]$ be a complete probability space and an increasing family of sub- $\sigma$ algebra of $\mathcal{F}$ and let $G \subset R^{2}$ be a bounded open set with the sufficiently smooth boundary $\Gamma=\Gamma_{0} \cup \Gamma_{1}$. The system equations in this paper are treated in the following function spaces

$$
V=H^{1}(G) \subset H=L^{2}(G) \subset V^{*}(\text { dual of } V)
$$

and

$$
\widetilde{V}=H^{\frac{1}{2}}\left(\Gamma_{1}\right) \subset \widetilde{H}=L^{2}\left(\Gamma_{1}\right) \subset \tilde{V}^{*}(\text { dual of } \tilde{V})
$$

where $H^{\alpha}(X)$ is a Sobolev space [4] of the order $\alpha$ on $X$.

We denote the norms in $H$ and $V$ by $|\cdot|$ and $\|\cdot\|$ respectively. $(\cdot, \cdot)$ denotes the inner product in $H$ and $\langle\cdot, \cdot\rangle$ is a duality between $V$ and $V^{*}$. The norms in $\widetilde{H}, \widetilde{V}$ are denoted by $|\cdot|_{\widetilde{H}}$ and $\|\cdot\|_{\widetilde{V}}$ respectively. $(\cdot, \cdot)_{\Gamma_{1}}$ and $\langle\cdot, \cdot\rangle_{\Gamma_{1}}$ denote an inner product in $\widetilde{H}$ and a duality between $\widetilde{V}$ and $\widetilde{V}^{*}$ respectively.

\section{Mathematical Model of the System Dynamics}

Assume that the boundary $\Gamma$ is constructed in such a way that $\Gamma=\Gamma_{0} \cup \Gamma_{1}$ where $\Gamma_{0}$ is a seepage boundary and $\Gamma_{1}$ contacts with the other media. Let $u(t, x)$ and $\psi(x)$ be a pressure of the fluid in $G$ and atmospheric pressure of the outside of $\Gamma_{0}$ at the time $t$ and the position $x$ respectively.

Assuming that the flow of the fluid in $G$ is incompressible and nonrotational, we have

$$
\frac{\partial^{2} u(t, x)}{\partial x^{2}}=0 \quad(t, x) \in \Theta \times G
$$


where $\Theta=(0, T)$.

Consider the boundary conditions associated with (3).

(1) On the boundary $\Gamma_{0}:$ There exist two situations;

(a) Because of a capillary action, the pressure of the fluid on this boundary $\Gamma_{0}$ may be less than the outside pressure, i.e., the atmospheric pressure $\psi(x)$. In this case, there is no flow across the boundary $\Gamma_{0}$, so we have

$$
\frac{\partial u(t, x)}{\partial \nu}=0 \text { if } u(t, x)<\psi(x) \text { on } \Theta \times \Gamma_{0}
$$

where $\nu$ is the normal at $\Gamma$ exterior to $G$.

(b) In the case where the pressure of the fluid is greater than or equal to the atmospheric pressure $\psi(x)$ on the boundary $\Gamma_{0}$, the fluid may seep out through the boundary $\Gamma_{0}$. It is reasonable to assume that the velocity of this outflow is proportional to the difference in the pressure, i.e., we have

$$
\begin{gathered}
\frac{\partial u(t, x)}{\partial \nu}=k(\psi(x)-u(t, x)) \text { if } u(t, x) \geq \psi(x) \\
\text { on } \Theta \times \Gamma_{0}
\end{gathered}
$$

where $k$ is a constant which denotes the permeability of the boundary $\Gamma_{0}$.

(2) On the boundary $\Gamma_{1}$ : We assume that the fluid can freely go in and out through the boundary $\Gamma_{1}$. Then, as seen in the percolation problem [1], we have

$$
\frac{\partial u(t, x)}{\partial t}+\frac{\partial u(t, x)}{\partial \nu}=f(t, x) \text { on } \Theta \times \Gamma_{1}
$$

with

$$
u(0, x)=u_{0}(x) \text { on } \Gamma_{1}
$$

where $f(t, x)$ denotes the interactive influence between the fluid in the porous media $G$ and the outside fluid at the boundary $\Gamma_{1}$.

It should be noted that the interactive influence changes randomly by the nonhomogeneous property of the outside media and impurities contained in the media. In this paper, this randomness is modelled by the white noise $d w(t) / d t$. Instead of (6), consider

$$
\begin{gathered}
\frac{\partial u(t, x)}{\partial t}+\frac{\partial u(t, x)}{\partial \nu}=f(t, x)+b(x) \frac{d w(t)}{d t} \\
\text { on } \Theta \times \Gamma_{1}
\end{gathered}
$$

where $w(t)$ is an $\mathcal{F}_{t}$-adapted standard Brownian motion process.

Define for any $\phi_{1}, \phi_{2} \in V$,

$$
\left\langle A \phi_{1}, \phi_{2}\right\rangle=\left(\frac{\partial \phi_{1}}{\partial x}, \frac{\partial \phi_{2}}{\partial x}\right) .
$$

Consider the stochastic variational form associated with (3) (5) and (8) with (7);

$$
\begin{gathered}
\int_{0}^{t}\langle A u(s), \phi\rangle d s+\int_{0}^{t}(\Psi(u(s)), \phi)_{\Gamma_{0}} d s \\
-\int_{0}^{t}\left\langle\frac{\partial u(s)}{\partial \nu}, \phi\right\rangle_{\Gamma_{1}} d s=0 \quad{ }^{\forall} \phi \in V
\end{gathered}
$$

and

$$
\begin{aligned}
& (u(t), \phi)_{\Gamma_{1}}+\int_{0}^{t}\left\langle\frac{\partial u}{\partial \nu}, \phi\right\rangle_{\Gamma_{1}} d s-\int_{0}^{t}(f, \phi)_{\Gamma_{1}} d s \\
& =\left(u_{0}, \phi\right)_{\Gamma_{1}}+\int_{0}^{t}(b, \phi)_{\Gamma_{1}} d w(s) \quad{ }^{\forall} \phi \in \tilde{V}
\end{aligned}
$$

where

$$
\Psi(\lambda)=\left\{\begin{array}{cc}
0, & \text { for } \lambda<\psi \\
k(\lambda-\psi), & \text { for } \lambda \geq \psi
\end{array}\right.
$$

Lemma 1 For a given function $h \in \widetilde{V}$ and $\psi \in$ $L^{2}\left(\Gamma_{0} ; R_{+}^{1}\right)$, there exists a unique function $v \in V$ which satisfies

$$
\langle A v, \phi\rangle+(\Psi(v), \phi)_{\Gamma_{0}}-\left\langle\frac{\partial v}{\partial \nu}, \phi\right\rangle_{\Gamma_{1}}=0 \quad{ }^{\forall} \phi \in V
$$

and

$$
v=h \quad \text { on } \Gamma_{1} .
$$

For the proof, see Appendix A.

Definition 1 From Lemma 1, the solution $v$ of (11) and (12) satisfies $\left.\frac{\partial v}{\partial \nu}\right|_{\Gamma_{1}} \in \widetilde{V}^{*}$. Therefore, we can define the oerator $\mathcal{B} \in \mathcal{L}\left(\tilde{V}, \widetilde{V}^{*}\right)$ in such a way that

$$
\mathcal{B} h=\frac{\partial v}{\partial \nu} \quad \text { on } \Gamma_{1}
$$

Lemma 2 The operator $\mathcal{B}$ defined by (13) is coercive in $\widetilde{V}$, i.e., the following inequality holds

$$
\langle\mathcal{B} h, h\rangle_{\Gamma_{1}} \geq \alpha\|h\|_{\widetilde{V}}^{2}, \quad{ }^{\exists} \alpha>0,{ }^{\forall} h \in \widetilde{V}
$$

Proof: Taking $\phi=v$ in (11) and noting $(\Psi(v), v)_{\Gamma_{1}} \geq 0$ and the continuity of the trace 
$V \rightarrow \widetilde{V}$, there exists $\alpha>0$ such that

$$
\begin{aligned}
\langle\mathcal{B} h, h\rangle_{\Gamma_{1}} & =\left(\frac{\partial v}{\partial \nu}, v\right)_{\Gamma_{1}} \\
& \geq\|v\|^{2} \\
& \geq \alpha\|v\|_{\widetilde{V}}^{2} \\
& =\alpha\|h\|_{\widetilde{V}}^{2} .
\end{aligned}
$$

Then, by using the opertor $\mathcal{B}$, the system (9) and (10) are rewritten by

$$
\begin{gathered}
\int_{0}^{t}\langle A u(s), \phi\rangle d s+\int_{0}^{t}(\Psi(u), \phi)_{\Gamma_{0}} d s \\
-\int_{0}^{t}\langle\mathcal{B} u(s), \phi\rangle_{\Gamma_{1}} d s=0 \quad{ }^{\forall} \phi \in V
\end{gathered}
$$

and

$$
\begin{gathered}
(u(t), \phi)_{\Gamma_{1}}+\int_{0}^{t}\langle\mathcal{B} u(s), \phi\rangle_{\Gamma_{1}} d s=\left(u_{0}, \phi\right)_{\Gamma_{1}} \\
+\int_{0}^{t}(f(s), \phi)_{\Gamma_{1}} d s+\int_{0}^{t}(b, \phi)_{\Gamma_{1}} d w(s) \\
{ }^{\forall} \phi \in \tilde{V}
\end{gathered}
$$

Theorem 1 With conditions

$$
\begin{array}{ll}
(C-1): & \psi \in L^{2}\left(\Gamma_{0} ; R_{+}^{1}\right) \\
(C-2): & u_{0} \in L^{2}(\Omega ; \widetilde{H}) \\
(C-3): & f \in L^{2}\left(\Theta ; \widetilde{V}^{*}\right) \\
(C-4): & b \in \widetilde{H},
\end{array}
$$

there exists a unique $\mathcal{F}_{t}$-adapted solution of (15) and (16) such that

$$
u \in L^{2}(\Omega \times \Theta ; V)
$$

and

$$
\left.u\right|_{\Gamma_{1}} \in L^{2}\left(\Omega ; L^{2}(\Theta ; \tilde{V}) \cap C(\bar{\Theta} ; \tilde{H})\right) .
$$

For the proof, see Appendix B.

\section{Boundary Observation Mech- anism}

Consider the following boundary observation mechanism

$$
y(t)=\int_{0}^{t} H u(s) d s+\int_{0}^{t} r(s) d e(s)
$$

where

$$
H(\cdot)=\int_{\Gamma_{1}} h(x)(\cdot) d x
$$

and $r, r^{-1} \in L^{\infty}(\Theta)$ and $e(t)$ is a standard Brownian motion process independent of the system noise $w(t)$.

Lemma 3 With the same conditions as in Theorem 1 and

(C-5): $r, r^{-1} \in L^{\infty}(\Theta), \quad h \in L^{\infty}\left(\Gamma_{1}\right)$, there exists a unique observation process $y(t)$ such that

$$
y \in L^{2}\left(\Omega ; C\left(\bar{\Theta} ; R^{1}\right)\right) .
$$

\section{Derivation of Estimator Dy- namics}

From Lemma 3, by using the nonlinear estimation theory[5], we have the following theorem.

Theorem 2 With conditions $(C-1) \sim(C-5)$, the optimal estimates $(\hat{u}(t), \phi)$ and $(\hat{u}(t), \phi)_{\Gamma_{1}}$ in the sense of the minimal variance estimate satisfy

$$
\begin{gathered}
\int_{0}^{t}\langle A \hat{u}(s), \phi\rangle d s+\int_{0}^{t}(\hat{\Psi}(u), \phi)_{\Gamma_{0}} d s \\
-\int_{0}^{t}\langle\mathcal{B} \hat{u}(s), \phi\rangle_{\Gamma_{1}} d s=0 \quad{ }^{\forall} \phi \in V, \\
(\hat{u}(t), \phi)_{\Gamma_{1}}+\int_{0}^{t}\langle\mathcal{B} \hat{u}(s), \phi\rangle_{\Gamma_{1}} d s \\
=\left(E\left(u_{0}\right), \phi\right)_{\Gamma_{1}}+\int_{0}^{t}(f(s), \phi)_{\Gamma_{1}} d s \\
+\int_{0}^{t} r^{-1}(s) H P_{\Gamma_{1}}(s) \phi d \ell(s) \\
{ }^{\forall} \phi \in \widetilde{V},
\end{gathered}
$$

$$
\begin{aligned}
& \int_{0}^{t}\left\langle A P(s) \phi_{1}, \phi_{2}\right\rangle d s+\int_{0}^{t}\left\langle A P(s) \phi_{2}, \phi_{1}\right\rangle d s \\
&-\int_{0}^{t}\left\langle\mathcal{B} P_{\Gamma_{1}} \phi_{1}, \phi_{2}\right\rangle_{\Gamma_{1}} d s \\
&-\int_{0}^{t}\left\langle\mathcal{B} P_{\Gamma_{1}} \phi_{2}, \phi_{1}\right\rangle_{\Gamma_{1}} d s \\
&+\int_{0}^{t} E\left\{\left(\Psi(u), \phi_{1}\right)_{\Gamma_{0}}\left(u-\hat{u}, \phi_{2}\right)_{\Gamma_{1}} \mid \mathcal{Y}_{s}\right\} d s \\
&+\int_{0}^{t} E\left\{\left(\Psi(u), \phi_{2}\right)_{\Gamma_{0}}\left(u-\hat{u}, \phi_{1}\right)_{\Gamma_{1}} \mid \mathcal{Y}_{s}\right\} d s=0 \\
& \forall \phi_{1}, \phi_{2} \in V
\end{aligned}
$$


and

$$
\begin{gathered}
\left(P_{\Gamma_{1}}(t) \phi_{1}, \phi_{2}\right)_{\Gamma_{1}}+\int_{0}^{t}\left\langle\mathcal{B} P_{\Gamma_{1}} \phi_{1}, \phi_{2}\right\rangle_{\Gamma_{1}} d s \\
+\int_{0}^{t}\left\langle\mathcal{B} P_{\Gamma_{1}} \phi_{2}, \phi_{1}\right\rangle_{\Gamma_{1}} d s \\
=\left(P_{\Gamma_{1}}(0) \phi_{1}, \phi_{2}\right)_{\Gamma_{1}}+\int_{0}^{t}\left(b, \phi_{1}\right)_{\Gamma_{1}}\left(b, \phi_{2}\right)_{\Gamma_{1}} d s \\
-\int_{0}^{t} r^{-2}(s) H P_{\Gamma_{1}}(s) \phi_{1} H P_{\Gamma_{1}}(s) \phi_{2} d s \\
+\int_{0}^{t} r^{-1} E\left\{H(u-\hat{u})\left(u-\hat{u}, \phi_{1}\right)_{\Gamma_{1}}\left(u-\hat{u}, \phi_{2}\right)_{\Gamma_{1}} \mid \mathcal{Y}_{s}\right\} d \ell \\
{ }^{\forall} \phi_{1}, \phi_{2} \in \tilde{V}
\end{gathered}
$$

where

$$
\begin{gathered}
P(t)(\cdot)=E\left\{u(u-\hat{u}, \cdot)_{\Gamma_{1}} \mid \mathcal{Y}_{t}\right\}, \\
P_{\Gamma_{1}}(t)(\cdot)=E\left\{u_{\Gamma_{1}}(u-\hat{u}, \cdot)_{\Gamma_{1}} \mid \mathcal{Y}_{t}\right\}, \\
\hat{u}(t)=E\left\{u(t) \mid \mathcal{Y}_{t}\right\}, \quad \mathcal{Y}_{t}=\sigma\{y(s) ; 0 \leq s \leq t\}
\end{gathered}
$$

and

$$
d \ell(t)=r^{-1}(t)(d y(t)-H \hat{u}(t) d t)
$$

It follows from (15) and (16) that

$$
\begin{aligned}
& (u(t), \phi)_{\Gamma_{1}}+\int_{0}^{t}\langle A u, \phi\rangle d s+\int_{0}^{t}(\Psi(u), \phi)_{\Gamma_{0}} d s \\
= & \left(u_{0}, \phi\right)_{\Gamma_{1}}+\int_{0}^{t}(f, \phi)_{\Gamma_{1}} d s+\int_{0}^{t}(b, \phi)_{\Gamma_{1}} d w .
\end{aligned}
$$

By applying the nonlinear filtering theory [5] to (25), Theorem 2 is derived.

Since the estimator dynamics derived in Theorem 2 is nonlinear, we introduce the following approximations,

$$
\Psi(u) \simeq \Psi(\hat{u})
$$

and

$$
E\left\{H(u-\hat{u})\left(u-\hat{u}, \dot{\phi_{1}}\right)_{\Gamma_{1}}\left(u-\hat{u}, \phi_{2}\right)_{\Gamma_{1}} \mid \mathcal{Y}_{s}\right\} \simeq 0 .
$$

By using approximations (26) and (27) to Theorem 2 , we have the following approximate estimator dynamics (still denoted the approximate estimate by $\hat{u}(t))$

$$
\begin{gathered}
\int_{0}^{t}\langle A \hat{u}(s), \phi\rangle d s+\int_{0}^{t}(\Psi(\hat{u}), \phi)_{\Gamma_{0}} d s \\
-\int_{0}^{t}\langle\mathcal{B} \hat{u}(s), \phi\rangle_{\Gamma_{1}} d s=0 \quad{ } \phi \in V
\end{gathered}
$$

$$
\begin{gathered}
(\hat{u}(t), \phi)_{\Gamma_{1}}+\int_{0}^{t}\langle\mathcal{B} \hat{u}(s), \phi\rangle_{\Gamma_{1}} d s \\
=\left(E\left(u_{0}\right), \phi\right)_{\Gamma_{1}}+\int_{0}^{t}(f(s), \phi)_{\Gamma_{1}} d s \\
+\int_{0}^{t} r^{-1}(s) H P_{\Gamma_{1}}(s) \phi d \ell(s) .{ }^{\forall} \phi \in \tilde{V}, \\
\int_{0}^{t}\left\langle A P(s) \phi_{1}, \phi_{2}\right\rangle d s+\int_{0}^{t}\left\langle A P(s) \phi_{2}, \phi_{1}\right\rangle d s \\
-\int_{0}^{t}\left\langle\mathcal{B} P_{\Gamma_{1}} \phi_{1}, \phi_{2}\right\rangle_{\Gamma_{1}} d s \\
-\int_{0}^{t}\left\langle\mathcal{B} P_{\Gamma_{1}} \phi_{2}, \phi_{1}\right\rangle_{\Gamma_{1}} d s=0
\end{gathered}
$$

and

$$
\left(P_{\Gamma_{1}}(t) \phi_{1}, \phi_{2}\right)_{\Gamma_{1}}+\int_{0}^{t}\left\langle\mathcal{B} P_{\Gamma_{1}} \phi_{1}, \phi_{2}\right\rangle_{\Gamma_{1}} d s
$$$$
+\int_{0}^{t}\left\langle\mathcal{B} P_{\Gamma_{1}} \phi_{2}, \phi_{1}\right\rangle_{\Gamma_{1}} d s
$$

$$
\begin{gathered}
=\left(P_{\Gamma_{1}}(0) \phi_{1}, \phi_{2}\right)_{\Gamma_{1}}+\int_{0}^{t}\left(b, \phi_{1}\right)_{\Gamma_{1}}\left(b, \phi_{2}\right)_{\Gamma_{1}} d s \\
-\int_{0}^{t} r^{-2}(s) H P_{\Gamma_{1}}(s) \phi_{1} H P_{\Gamma_{1}}(s) \phi_{2} d s .
\end{gathered}
$$

\section{Numerical Example}

Consider the following one dimensional system;

$$
\frac{\partial^{2} u(t, x)}{\partial x^{2}}=0 \quad 0<t<T, \quad 0<x<1
$$

with the boundary conditions

$$
\frac{\partial u(t, 0)}{\partial x}=\left\{\begin{array}{cc}
0, & \text { if } u(t, 0)<\psi \\
k(u(t, 0)-\psi), & \text { if } u(t, 0) \geq \psi
\end{array}\right.
$$

and

$$
\frac{\partial u(t, 1)}{\partial t}+a \frac{\partial u(t, 1)}{\partial x}=f(t)+b \frac{d w(t)}{d t}
$$

with

$$
u(0,1)=u_{0} .
$$

The boundary observation mechanism is given by

$$
y(t)=\int_{0}^{t} h u(s, 1) d s+\int_{0}^{t} r d e(s) .
$$


Then, the approximate estimator dynamics becomes

$$
\frac{\partial^{2} \hat{u}(t, x)}{\partial x^{2}}=0 \quad 0<t<T, 0<x<1
$$

with the boundary conditions

$$
\frac{\partial \hat{u}(t, 0)}{\partial x}=\left\{\begin{array}{cc}
0, & \text { if } \hat{u}(t, 0)<\psi \\
k(\hat{u}(t, 0)-\psi), & \text { if } \hat{u}(t, 0) \geq \psi
\end{array}\right.
$$

and

$$
\begin{aligned}
\frac{\partial \hat{u}(t, 1)}{\partial t} & +a \frac{\partial \hat{u}(t, 1)}{\partial x}=f(t) \\
& +r^{-1} h p(t, 1,1) \frac{d \ell(t)}{d t}
\end{aligned}
$$

with

$$
\hat{u}(0,1)=E\left\{u_{0}\right\}
$$

where $p(t, x, 1)$ is the kernel of $P(t)$.

With the following parameters, simulation experiments are performed:

$a=4 ; b=0.15 ; h=4 ; r=0.4 ; k=0.25 ; u_{0}=$ $1.2 ; \psi=1 ; f=0.025 \sin (2 \pi t) ; P(0)=0.3, E\left\{u_{0}\right\}=$ 0 .

Sample runs of the true and estimate states at $x=1$ are shown in Fig.1.

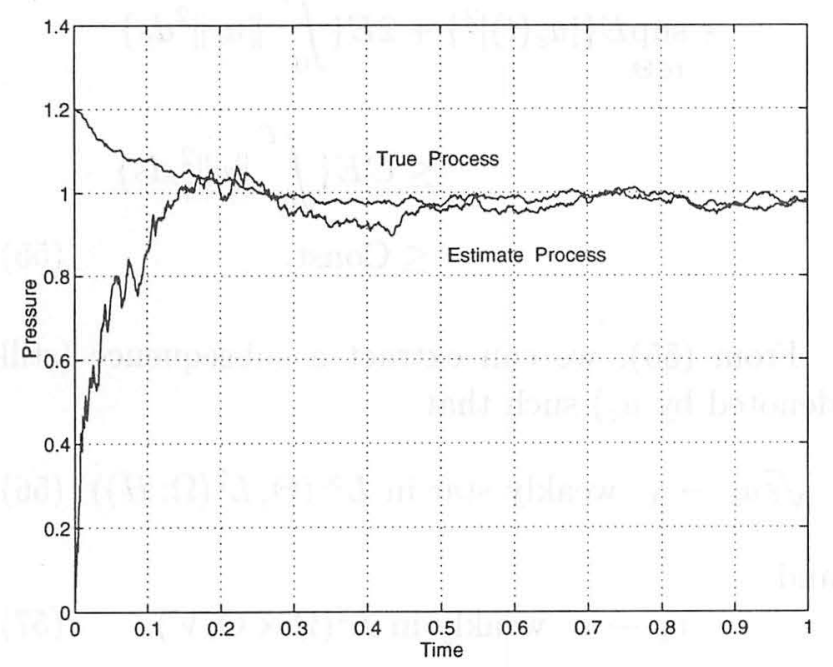

Fig.1 Sample Runs of True and Estimate Processes

\section{Conclusions}

In this paper, the state estimation in the filtration problem has been considered under the noisy boundary observation. Because of the existence of the nonlinear boundary condition, the estimator dynamics derived here becomes nonlinear. Then, a simple but useful approximation for the nonlineat estimator dynamics was proposed and the efficiency of the approximation was shown through the simulation experiments. The analytical method for proving the existence of the unique solution to the stochastic partial differential equations with the dynamic boundary condition will be effective for the other type of filtration problems. This research is partially supported by the Japan Ministry of Education under Grant C-(2)07650286.

\section{References}

[1] A. Friedman: Variational Principles and Free Boundary Problems, Jhon-Wiley \& Sons, (1982)

[2] C. M. Elliott and A. Friedman: Analysis of a Model of Percolation in a Gently Sloping Sand-Bank, SIAM J. Math. Anal., 165,941/954 (1985)

[3] J.L. Lions: Optimal Contro Governed by Partial Differential Equations, Springer (1971)

[4] R.A. Adams: Sobolev Spaces, Academic Press (1975)

[5] R.S. Liptser and A.N. Shiryayev: Statistics and Random Processes I, Springer (1978)

\section{Appendices}

The proof is performed by using Galerkin's approximation method, however, for simplicity, the part of the approximation is omitted in Appendices A and B.

\section{A Proof of Lemma 1}

Noting that for a given function $h \in \tilde{V}$, there exists an extension $\widetilde{h} \in V$ such that

$$
\left.\widetilde{h}\right|_{\Gamma_{1}}=h,\left.\quad \widetilde{h}\right|_{\Gamma_{0}}=0 .
$$

Define

$$
\widetilde{v}=v-\tilde{h} \text { in } G
$$

Noting that $\widetilde{v}=0$ on $\Gamma_{1}$, we introduce

$$
W=\left\{\phi|\phi \in V, \quad \phi|_{\Gamma_{1}}=0\right\}
$$


Then, from $\widetilde{v}=v$ on $\Gamma_{0},(11)$ is rewritten by

$$
\langle A \widetilde{v}, \phi\rangle+(\Psi(\widetilde{v}), \phi)_{\Gamma_{0}}+\langle A \widetilde{h}, \phi\rangle=0 \quad{ }^{\forall} \phi \in W
$$

In (43), taking $\phi=\widetilde{v}$, we have

$$
\langle A \widetilde{v}, \widetilde{v}\rangle+(\Psi(\widetilde{v}), \widetilde{v})_{\Gamma_{0}}+\langle A \widetilde{h}, \widetilde{v}\rangle=0
$$

It follows from the definition of $\Psi$ and $\psi \geq 0$ that

$$
(\Psi(\widetilde{v}), \widetilde{v})_{\Gamma_{0}} \geq 0 .
$$

Noting (45), from (43), we have

$$
\|\widetilde{v}\|^{2} \leq \frac{\|\widetilde{v}\|^{2}}{2}+\frac{\|\widetilde{h}\|^{2}}{2} .
$$

Therefore, we have

$$
\|\widetilde{v}\|^{2} \leq\|\widetilde{h}\|^{2} \leq \text { Const. }
$$

(41), (42) and (46) yield

$$
v \in V,\left.\quad v\right|_{\Gamma_{1}}=h
$$

\section{B Proof of Theorem 1}

Consider

$$
\begin{aligned}
& (v(t), \phi)_{\Gamma_{1}}+\int_{0}^{t}\langle\mathcal{B} v, \phi\rangle_{\Gamma_{1}} d s=\left(u_{0}, \phi\right)_{\Gamma_{1}} \\
+ & \int_{0}^{t}(f, \phi)_{\Gamma_{1}} d s+\int_{0}^{t}(b, \phi)_{\Gamma_{1}} d s \quad{ }^{\forall} \phi \in \widetilde{V}
\end{aligned}
$$

Under the Gelfand triple (2), since the operator $\mathcal{B}$ is coercive in $\widetilde{V}$ from Lemma 2 , it is easily shown that there exists a unique solution $v$ of (48) such that

$$
v \in L^{2}\left(\Omega ; C(\bar{\Theta} ; H) \cap L^{2}(\bar{\Theta} ; \tilde{V})\right) .
$$

Therefore, (15) and (16) are interpreted by

$$
\begin{aligned}
\int_{0}^{t}\langle A u, \phi\rangle & d s+\int_{0}^{t}(\Psi(u), \phi)_{\Gamma_{0}} d s \\
- & \int_{0}^{t}\langle\mathcal{B} u, \phi\rangle_{\Gamma_{1}} d s=0 \quad{ }^{\forall} \phi \in V
\end{aligned}
$$

and

$$
\left.u\right|_{\Gamma_{1}}=v \in L^{2}\left(\Omega ; C(\bar{\Theta} ; H) \cap L^{2}(\bar{\Theta} ; \tilde{V})\right) .
$$

Consider the approximate equation associated with (50) and (51) for ${ }^{\forall} \varepsilon>0$,

$$
\varepsilon\left(u_{\varepsilon}(t), \phi\right)+\int_{0}^{t}\left\langle A u_{\varepsilon}, \phi\right\rangle d s+\int_{0}^{t}\left(\Psi\left(u_{\varepsilon}\right), \phi\right)_{\Gamma_{0}} d s
$$

$$
-\int_{0}^{t}\left\langle\mathcal{B} u_{\varepsilon}, \phi\right\rangle_{\Gamma_{1}} d s=0 \quad{ }^{\forall} \phi \in V
$$

and

$$
\left.u_{\varepsilon}\right|_{\Gamma_{1}}=v \in L^{2}\left(\Omega ; C(\bar{\Theta} ; H) \cap L^{2}(\bar{\Theta} ; \tilde{V})\right) .
$$

It follows from Ito's lemma that

$$
\begin{aligned}
\varepsilon\left|u_{\varepsilon}(t)\right|^{2} & +2 \int_{0}^{t}\left\langle A u_{\varepsilon}, u_{\varepsilon}\right\rangle d s \\
& +2 \int_{0}^{t}\left(\Psi\left(u_{\varepsilon}\right), u_{\varepsilon}\right)_{\Gamma_{0}} d s \\
& -2 \int_{0}^{t}\left\langle\mathcal{B} u_{\varepsilon}, u_{\varepsilon}\right\rangle_{\Gamma_{1}} d s=0
\end{aligned}
$$

Noting that

$$
\begin{aligned}
2\left\langle\mathcal{B} u_{\varepsilon}, u_{\varepsilon}\right\rangle_{\Gamma_{1}} & \leq\left\|\mathcal{B} u_{\varepsilon}\right\|_{\widetilde{V}^{*}}^{2}+\left\|u_{\varepsilon}\right\|_{\widetilde{V}}^{2} \\
& \leq C\left\|u_{\varepsilon}\right\|_{\widetilde{V}}^{2} \\
& =C\|v\|_{\widetilde{V}}^{2}
\end{aligned}
$$

and

$$
\left(\Psi\left(u_{\varepsilon}\right), u_{\varepsilon}\right)_{\Gamma_{1}} \geq 0
$$

we have

$$
\begin{aligned}
\varepsilon \sup _{t \in \bar{\Theta}} E\left\{\left|u_{\varepsilon}(t)\right|^{2}\right\} & +2 E\left\{\int_{0}^{T}\left\|u_{\varepsilon}\right\|^{2} d s\right\} \\
& \leq C E\left\{\int_{0}^{T}\|v\|_{\widetilde{V}}^{2} d s\right\} \\
& \leq \text { Const. }
\end{aligned}
$$

From (55), we can extract a subsequence (still denoted by $u_{\varepsilon}$ ) such that

$$
\sqrt{\varepsilon} u_{\varepsilon} \rightarrow \chi \text { weakly star in } L^{\infty}\left(\Theta ; L^{2}(\Omega ; H)\right)
$$

and

$$
u_{\varepsilon} \rightarrow u \text { weakly in } L^{2}(\Omega \times \Theta ; V) .
$$

It follows from (56) and (57) that

$$
\begin{gathered}
\int_{0}^{t}\langle A u, \phi\rangle d s+\int_{0}^{t}(\Psi(u), \phi)_{\Gamma_{0}} d s \\
-\int_{0}^{t}\langle\mathcal{B} u, \phi\rangle_{\Gamma_{1}} d s=0 \quad{ }^{\forall} \phi \in V .
\end{gathered}
$$

The uniqueness of the solution follows from the monotonicity property of $\Psi(\cdot)$. 